\title{
Multi-scale image fusion for X-ray grating-based mammography
}

\author{
Xiaolei Jiang ${ }^{\mathrm{a}, \mathrm{b}}$, Li Zhang ${ }^{\text {*a,b }}$, Zhentian Wang ${ }^{\mathrm{c}}$, Marco Stampanoni ${ }^{\mathrm{c}, \mathrm{d}}$ \\ ${ }^{\mathrm{a}}$ Department of Engineering Physics, Tsinghua University, Beijing, 100084, China; \\ ${ }^{\mathrm{b}}$ Key Laboratory of Particle \& Radiation Imaging (Tsinghua University), Ministry of Education, \\ Beijing, 100084, China; \\ ${ }^{\mathrm{c}}$ Swiss Light Source, Paul Scherrer Institut, Villigen, 5232, Switzerland; \\ ${ }^{\mathrm{d}}$ Institute for Biomedical Engineering, University and ETH Zürich, Zürich, 8092, Switzerland
}

\begin{abstract}
X-ray phase contrast imaging (PCI) can provide high sensitivity of weakly absorbing low-Z objects in medical and biological fields, especially in mammography. Grating-based differential phase contrast (DPC) method is the most potential PCI method for clinic applications because it can works well with conventional X-ray tube and it can retrieve attenuation, DPC and dark-field information of the samples in a single scanning. Three kinds of information have different details and contrast which represent different physical characteristics of X-rays with matters. Hence, image fusion can show the most desirable characteristics of each image.

In this paper, we proposed a multi-scale image fusion for X-ray grating-based DPC mammography. Firstly, non-local means method is adopted for denoising due to the strong noise, especially for DPC and dark-field images. Then, Laplacian pyramid is used for multi-scale image fusion. The principal component analysis (PCA) method is used on the high frequency part and the spatial frequency method is used on the low frequency part. Finally, the fused image is obtained by inverse Laplacian pyramid transform. Our algorithm is validated by experiments. The experiments were performed on mammoDPC instrumentation at the Paul Scherrer Institut in Villigen, Switzerland. The results show that our algorithm can significantly show the advantages of three kinds of information in the fused image, which is very helpful for the breast cancer diagnosis.
\end{abstract}

Keywords: phase contrast, mammography, non-local means, PCA, spatial frequency, image fusion

\section{INTRODUCTION}

Hard X-ray phase contrast imaging (PCI) developed slowly until the third-generation synchrotron radiation sources were put into wide practice. Various hard X-ray phase-contrast imaging methods, such as the interferometer-based ${ }^{1}$, propagation-based $^{2}$, analyser-based ${ }^{3,4}$, and grating-based methods ${ }^{5,6}$, have been implemented to obtain highly sensitive phase contrast images, especially for low-Z samples. Basic experiments have made great success in the applications in various fields, including medicine, biology and chemistry, etc.

Among various PCI methods, hard X-ray grating-based imaging has the most potential for clinical applications. David et $\mathrm{al}^{5}$, Momose et $\mathrm{al}^{6}$ and Weitkamp et $\mathrm{al}^{7}$ firstly implemented the X-ray grating-based PCI method on synchrotron radiation sources. Their methods are based on the Talbot theory and they all rely on the highly coherence of synchrotron radiation sources. In 2006, Pfeiffer et $\mathrm{al}^{8}$ proposed a Talbot-Lau interferometry with three gratings to implement DPC imaging with low-brilliance X-ray sources shown as Fig.1. Two years later, they retrieved (ultra) small angle scattering information additionally under the similar systemic conFig ${ }^{9}$. On the other hand, our group proposed an alternative method based on classic optics theory under the incoherent condition to achieve the retrieval of multiple information by use of the phase-stepping approach ${ }^{10}$.

Three kinds of information, that is attenuation, DPC and dark-field images, can be obtained simultaneously in a single scanning, which represent different physical characteristics of X-rays with matters. In this paper, we focus on the fusion of three kinds of information. A multi-scale image fusion process is proposed. Non-local means method is adopted for denoising before image fusion due to the strong noise, especially for DPC and dark-field images. Then, Laplacian

*Li Zhang: E-mail: zli@mail.tsinghua.edu.cn

Developments in X-Ray Tomography VIII, edited by Stuart R. Stock,

Proc. of SPIE Vol. 8506, 850612 - (c) 2012 SPIE · CCC code: 0277-786/12/\$18

doi: $10.1117 / 12.929625$ 
pyramid is used for multi-scale image fusion. The principal component analysis (PCA) method is used on the high frequency part and the spatial frequency method is used on the low frequency part. Finally, the fused image is obtained by inverse Laplacian pyramid transform. The method is validated by actual experiments, which were performed on mammoDPC instrumentation at the Paul Scherrer Institut in Villigen, Switzerland. The results show that our method can significantly show the advantages of three kinds of information in the fused image, which is very helpful for the breast cancer diagnosis.

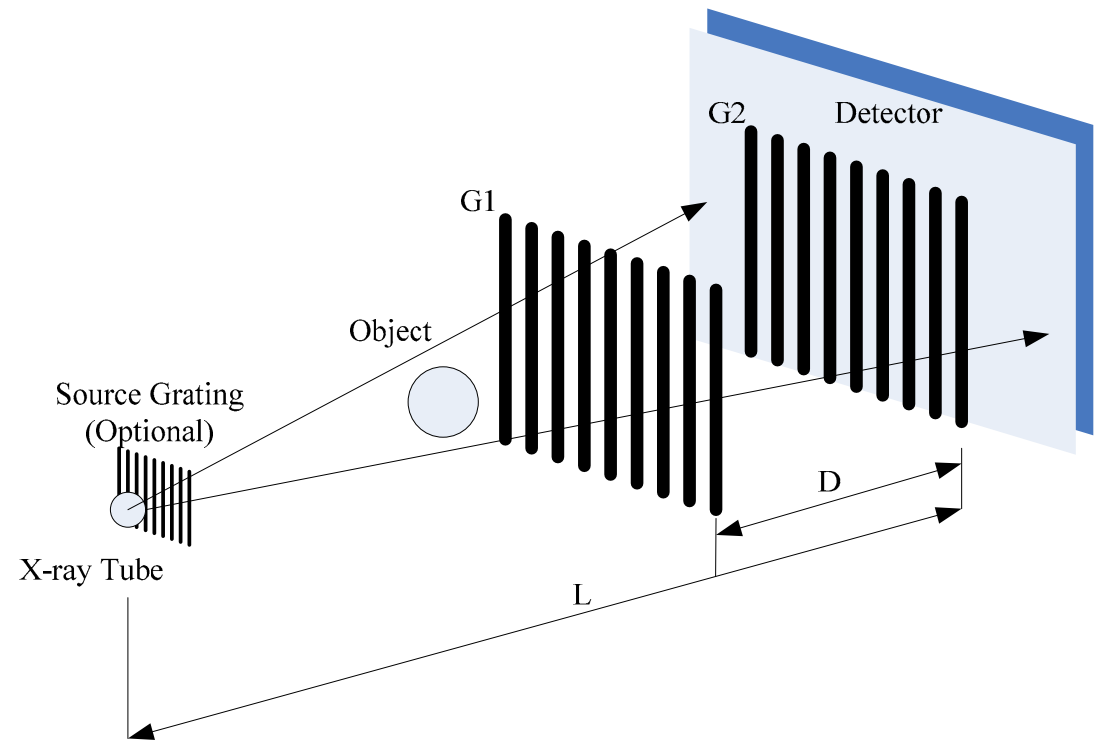

Fig.1. The schematic diagram of the grating-based imaging system.

\section{METHODOLOGY}

\subsection{Non-local means denoising method}

Many sources of uncertainties will affect the image qualities. Ref ${ }^{11,12}$ have analyzed the noise properties of grating-based imaging. Images are impacted by noises, especially for the DPC and dark-field images, which makes it difficult for image fusion. Hence, image denoising is essential before image fusion.

Recently, many denoising methods have been proposed to address the problem, among which the non-local means (NLM) denoising method is very powerful. Inspired by the self-similarity of natural images, Buades et $\mathrm{al}^{13}$ proposes the NLM denoising method based on the similarity between image patches. The NLM method estimates pixel intensity as a weighted average, and the weight measures the similarity between the pixel and the pixel to be denoised, prior to the additive noise degradation. Given a discrete noisy image $v=\{v(i) \mid i \in I\}$, the estimated value $N L(v)(i)$ can be computed as Eq.(7).

$$
N L(v)(i)=\sum_{j \in \Omega} w(i, j) v(j)
$$

where $\Omega$ represents the search volume, $w(i, j)$ is the weight that measures similarity between the pixels $i$ and $j$, which is associated with the quadratic distances shown as Eq.(8).

$$
w(i, j)=\frac{1}{Z(i)} e^{-\frac{\left\|N_{i}-N_{j}\right\|_{2}^{2}}{h^{2}}}
$$


where \|\|$_{2}$ denotes the Euclidean distance, $Z(i)$ is the normalizing factor $Z(i)=\sum_{j \in \Omega} e^{-\frac{\left\|N_{i}-N_{j}\right\|_{2}^{2}}{h^{2}}}$ and the parameter $h$ controls the decay of the exponential function and therefore the decay of the weights, which is associated with the noise variance $\sigma^{2}$.

\subsection{Image fusion}

\subsubsection{Laplacian pyramid decomposition}

The Laplacian pyramid (LP), originally proposed by Burt and Adelson ${ }^{14}$ before multiresolution wavelet analysis was introduced, is a bandpass image decomposition derived from the Gaussian pyramid (GP) which is a multi-resolution image representation obtained through a recursive reduction (lowpass filtering and decimation) of the image data set.

Suppose the zero level of the pyramid $G_{0}$ is equal to the source image, $G_{0}$ is on the bottom of the pyramid, and the $l$-th level of Gaussian pyramid which denoted as $G_{l}$ is obtained by those steps:

Firstly, the convolution is performed between the $l$-1-th level images $G_{l-1}$ and the window function $\omega(m, n)$ which has low-pass characteristics. Then, convolution results were separated out in the downsampling:

$$
G_{l}(i, j)=\sum_{m=-2}^{2} \sum_{n=-2}^{2} \omega(m, n) G_{l-1}(2 i+m, 2 j+n) \quad 1 \leq l \leq N, 0 \leq i<R_{b}, 0 \leq j<C_{l}
$$

where $N$ is the maximal level of pyramid, $R_{b}$ and $C_{l}$ are the column and row number of the $l$-th level pyramid resprectively, $\omega(m, n)$ is weighting function, which is a two-dimensional separable $5 \times 5$ window function:

$$
\omega=\frac{1}{256}\left(\begin{array}{ccccc}
1 & 4 & 6 & 4 & 1 \\
4 & 16 & 24 & 16 & 4 \\
6 & 24 & 36 & 24 & 6 \\
4 & 16 & 24 & 16 & 4 \\
1 & 4 & 6 & 4 & 1
\end{array}\right)
$$

Then the Gaussian pyramid is constituted by $G_{0}, G_{1}, \ldots, G_{N}$, where $G_{0}$ is the bottom and $G_{N}$ is the top of the pyramid, respectively. The Laplacian pyramid is derived from the Gaussian pyramid, which can find the difference between the adjacent two images and get the band-pass filtered images.

Let $G_{l}^{*}$ be the image obtained by expanding $G_{l}$, then $G_{l}^{*}$ has the same size with $G_{l-1}$.

$$
G_{l}^{*}(i, j)=4 \sum_{m=-2}^{2} \sum_{n=-2}^{2} \omega(m, n) G_{l}\left(\frac{i+m}{2}, \frac{j+n}{2}\right) \quad 1 \leq l \leq N, 0 \leq i<R_{b}, 0 \leq j<C_{l}
$$

Where

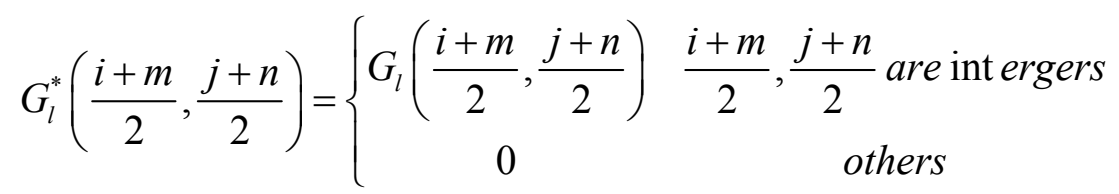

Set 


$$
\left\{\begin{array}{cc}
L P_{l}=G_{l}-G_{l+1}^{*}, & 0 \leq l<N \\
L P_{N}=G_{N}, & l=N
\end{array}\right.
$$

where $\mathrm{N}$ is the number of Laplacian pyramid levels, $L P_{l}$ is the $l$-th level image decomposed from Laplacian pyramid. Now the Laplacian pyramid composed of $L P_{0}, L P_{1}, \ldots, L P_{N}$ can be obtained, each of which is the difference of its Gaussian pyramid image itself and the last level's has been interpolated and enlarged

\subsubsection{Fusion strategy}

Laplacian pyramid represents the edge of the image detail at every levels, so by comparing the corresponding Laplacelevel pyramid of two images, it is possible to obtain the fused image which merge their respective outstanding details, and makes the integration of the image retaining the amount of information as rich as possible. The source image is decomposed into a series of resolution spaces, and how to choose integration factor and fusion rule will directly affect the final quality of fused image.

In order to reflect the tiny details and texture characteristics of the image, to different level of pyramid, different fusion operators are proposed.

(1) To the top level N, we adopt the spatial frequency (SF) method $^{15}$.

Spatial frequency measures the overall information level in an image ${ }^{16}$, and for an image $I$ of dimension $M \times N$, it's defined as follows:

Row frequency: $\quad R F=\sqrt{\frac{1}{M N} \sum_{i=0}^{M-1} \sum_{j=1}^{N-1}[I(i, j)-I(i, j-1)]^{2}}$

Column frequency:

$$
C F=\sqrt{\frac{1}{M N} \sum_{j=0}^{N-1} \sum_{i=1}^{N-1}[I(i, j)-I(i-1, j)]^{2}}
$$

Spatial frequency:

$$
S F=\sqrt{R F^{2}+C F^{2}}
$$

Here, $M$ is the number of rows, $N$ is the number of columns.

The SF-based weighted-image fusion is as follows:

The computed SFs are normalized as

$$
N S F_{1}=\frac{S F_{1}}{S F_{1}+S F_{2}} \text { and } N S F_{2}=\frac{S F_{2}}{S F_{1}+S F_{2}}
$$

The fused is obtained by

$$
I_{f}=N S F_{1} I_{1}+N S F_{2} I_{2}
$$

(2) To the rest levels $(0 \leq l \leq L)$, we adopt the principal component analysis (PCA) method $^{17}$.

The fusion using PCA is achieved by weighted sum of source images. The weights for each source image are obtained from the normalized Eigen vector of the covariance matrices of each source image.

We implement kernel based PCA the three input images, that is the attenuation, DPC and dark-field images. Covariance matrix and the corresponding normalized Eigen values for this kernel are calculated. Normalized Eigen values are applied as weights for the input images and the fused image is the weighted sum of input images.

\subsubsection{Laplacian pyramid reconstruction}

The Laplacian pyramid reconstruction is the inversion of the Laplacian pyramid decomposition. From (9), then 


$$
\left\{\begin{array}{c}
G_{N}=L P_{N}, \quad l=N \\
G_{l}=L P_{l}+G_{l+1}^{*}, \quad 0 \leq l<N
\end{array}\right.
$$

As the assumption, the source image is just $G_{0}$. We can recover the original image exactly.

\section{EXPERIMENT}

We validated the method using the mammography results. The experiments were carried out on the mammoDPC instrumentation at the Paul Scherrer Institut in Villigen, Switzerland, which is the first whole fresh breast grating-based imaging. The detailed parameters can be found in $\operatorname{Ref}^{18}$.

Three kinds of information, that is attenuation, DPC and dark-field images, and their regions of interest are shown in Fig. 2. DPC image and dark-field image can provide extra information. However, they are subject to strong noises, which make it difficult for the image fusion. Therefore, image denoising is quite essential before image fusion. The denoised results by NLM of three kinds of information are shown in Fig. 3. The denoising method is quite powerful for image denoising while preserving signal characteristics. The fused image by the proposed image fusion algorithm is shown in Fig. 4. The number of Laplacian pyramid levels is 4 . And PCA method is used on the high frequency part and the spatial frequency method is used on the low frequency part. The fused result can significantly show the advantages of three kinds of information in one image which is very helpful for doctors.

\section{CONCLUSION}

In this paper, a multi-scale image fusion for X-ray grating-based DPC mammography was proposed. Firstly, non-local means method was adopted for denoising. After that, Laplacian pyramid was used for multi-scale image fusion. The principal component analysis (PCA) method was used on the high frequency part and the spatial frequency method was used on the low frequency part. Finally, the fused image was obtained by inverse Laplacian pyramid transform. The algorithm was validated by mammography experiments. The results can significantly show the advantages of three kinds of information, which is very helpful for diagnosis.

\section{ACKNOWLEDGEMENTS}

The work was supported by a grant from the National Natural Science Foundation of China (NNSFC) grants 10905031 and 61171178 and the Specialized Research Fund for the Doctoral Program of Higher Education grant 20090002120016 and 20110490366. 


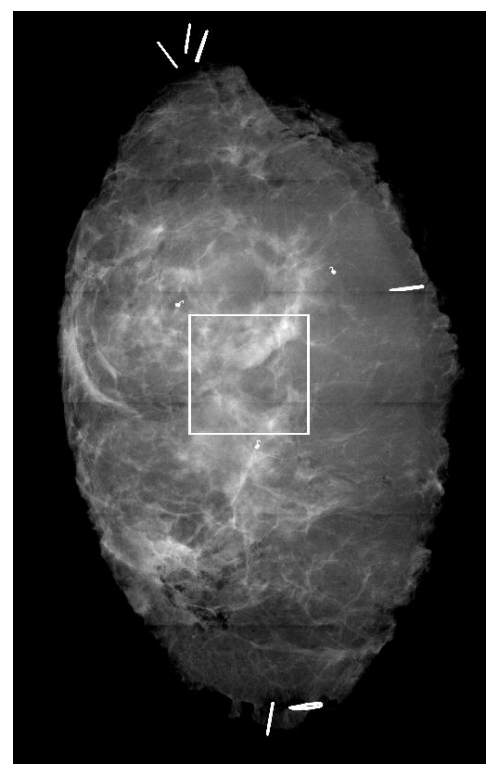

(a)

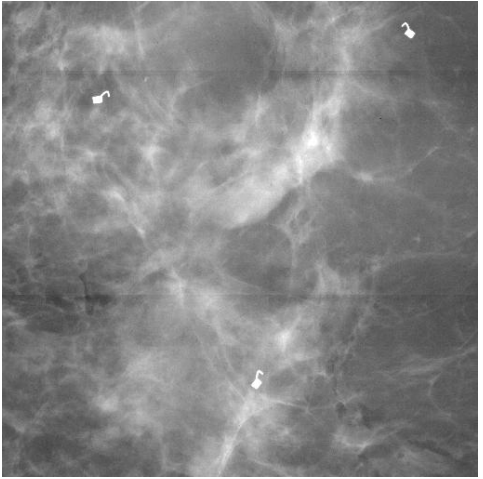

(d)

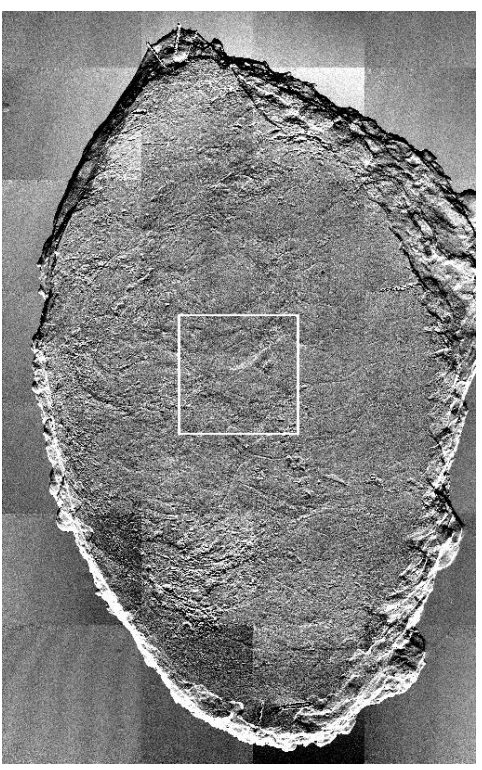

(b)

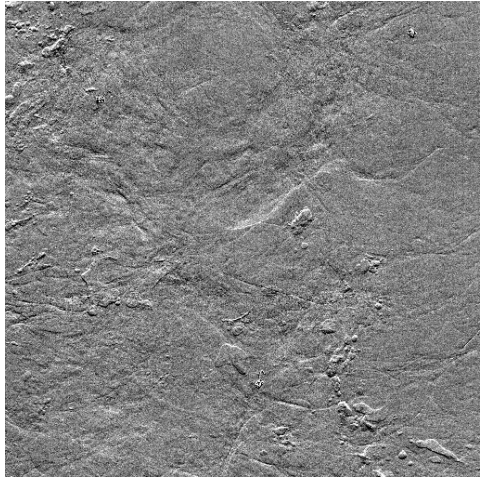

(e)

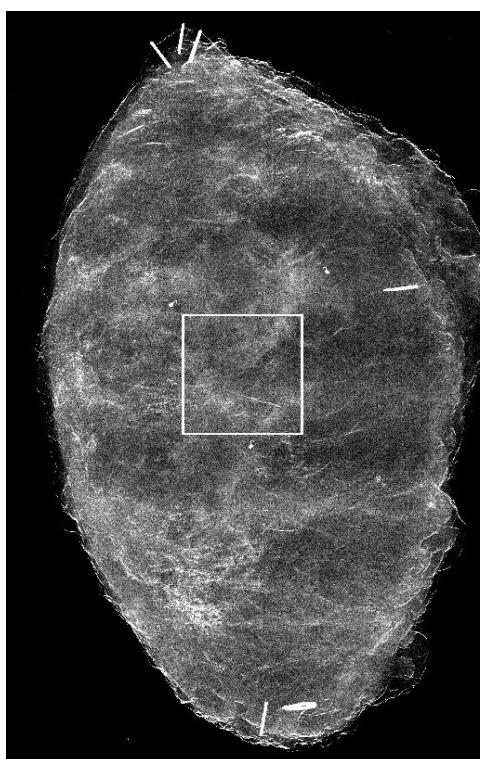

(c)

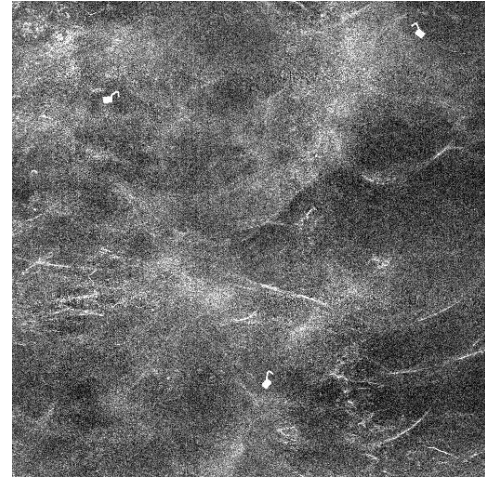

(f)

Fig.2 Three kinds of information: (a) Attenuation image; (b) DPC image; (c) Dark-field image. And their regions of interest: (d) Attenuation image; (e) Phase-contrast image; (f) Dark-field image.

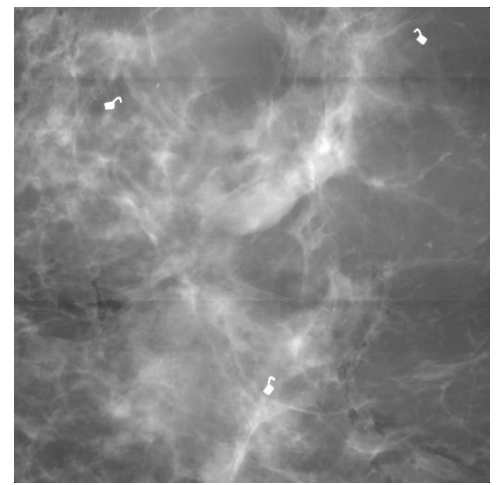

(a)

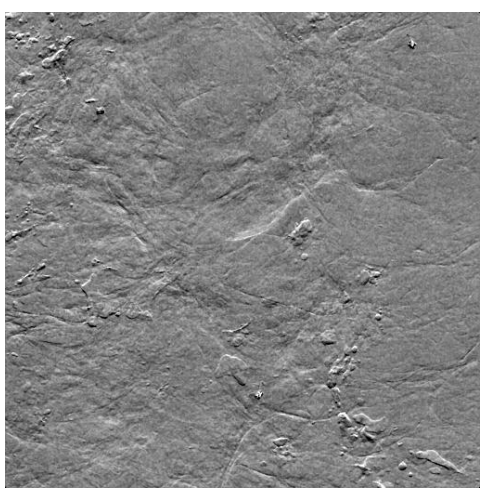

(b)

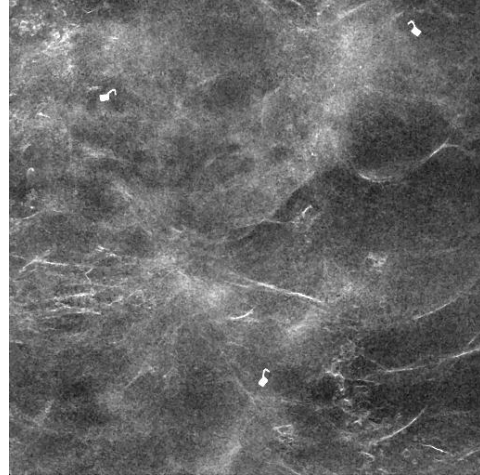

(c)

Fig. 3 The denoised results by NLM of three kinds of information: (a) Attenuation image; (b) DPC image; (c) Dark-field image. 


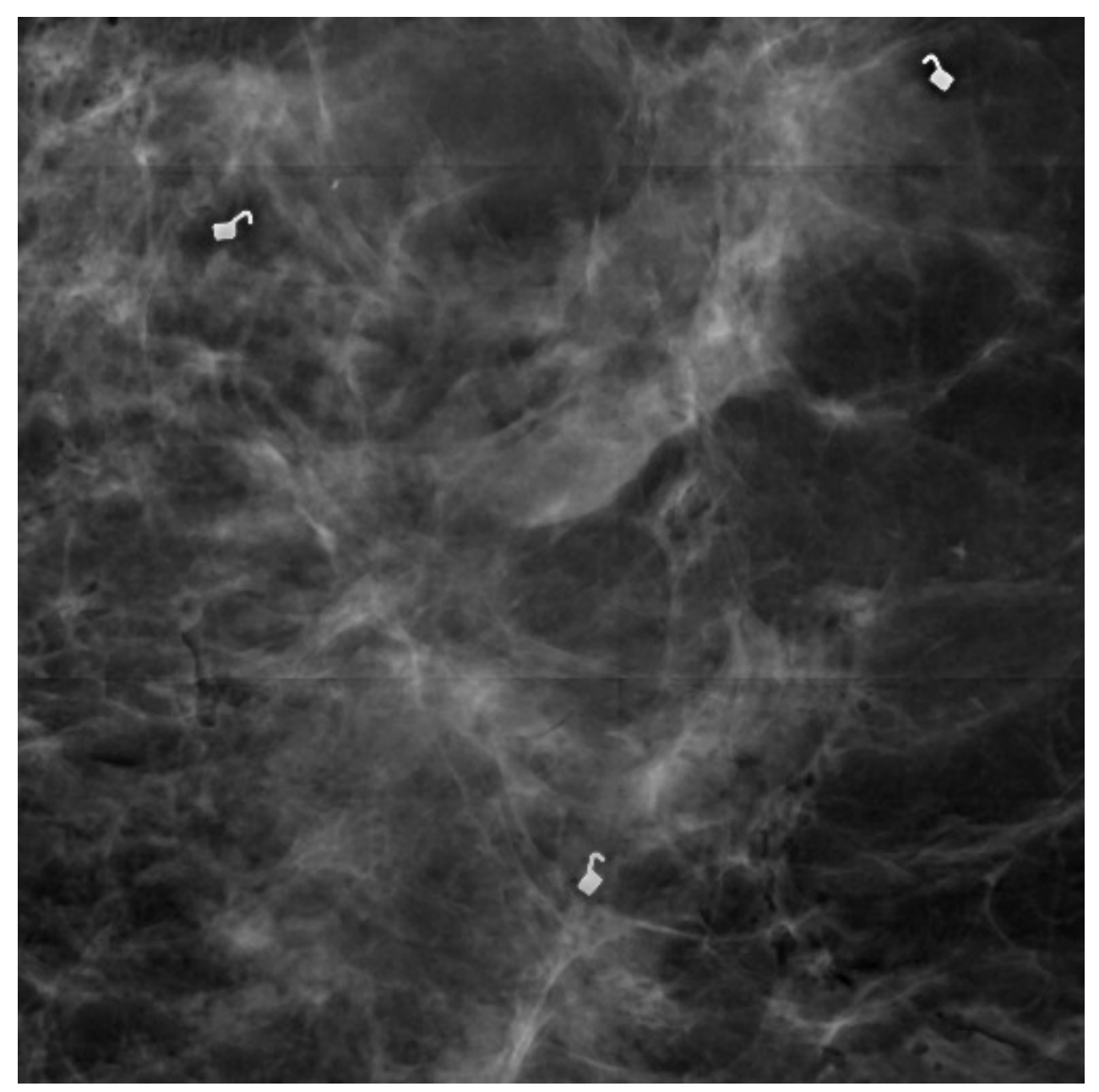

Fig.4 The fused image of attenuation, DPC and dark-field images.

\section{REFERENCES}

[1] Momose A., "Demonstration of phase-contrast X-ray computed tomography using an X-ray interferometer," Nuclear Instruments and Methods in Physics Research A, 352(3), 622-628 (1995).

[2] Snigirev A., Snigireva I., Kohn V., Kuznetsov S. and Schelokov I., "On the possibilities of x-ray phase contrast microimaging by coherent high-energy synchrotron radiation," Review of Scientific Instruments, 66(12), 5486$5492(1995)$

[3] Davis T., Gao D., Gueyev T., Stevenson A. and Wilkins S., "Phase-contrast imaging of weakly absorbing materials using hard X-rays," Nature, 373, 595-597 (1995).

[4] Chapman D., Thomlinson W., Johnston R., Washburn D., Pisano E., Gmur N., Zhong Z., Menk R., Arfelli F. and Sayers D., "Diffraction enhanced x-ray imaging. Physics in Medicine and Biology," Phys. Med. Biol., 42, 2015-2025 (1997). 
[5] David C., Nohammer B., Solak H H., "Differential x-ray phase contrast imaging using a shearing interferometer," Applied Physics Letters, 81(17), 3287-3289 (2002).

[6] Momose A, Kawamoto S, Koyama I. "Phase tomography using an x-ray Talbot interferometer," Proc. of SPIE, 5535, 352-360 (2004).

[7] Weitkamp T., Diaz A., Nohammer B., "Hard x-ray phase imaging and tomography with a grating interferometer," Proc. of SPIE, 5535, 352-360 (2004).

[8] Pfeiffer F., Weitkamp T., Bunk O., "Phase retrieval and differential phase-contrast imaging with low-brilliance X-ray sources," Nat Phys, 2(4), 258-261(2006).

[9] Pfeiffer F., Bech M., Bunk O., "Hard-X-ray dark-field imaging using a grating interferometer," Nat Mater, 7(2), 134-137 (2008).

[10] Huang Z F, Kang K J, Zhang L, "Alternative method for differential phase-contrast imaging with weakly coherent hard x rays," Physical Review A, 79(1), 013815-6 (2009).

[11] Revol V., Kottler C., Kaufmann R., Straumann U. and Urban C., "Noise analysis of grating-based x-ray differential phase contrast imaging," Review of Scientific Instruments 81, 073709 (2010).

[12] Jiang X L, Zhang L., Huang Z F and Zhang R., "Fast multiple information retrieval algorithm for X-ray gratingbased imaging," Proc. of IEEE NSS/MIC, 3429-3433 (2011)

[13] Buades A., Coll B., and Morel J., "A non-local algorithm for image denoising," Proc. IEEE CVPR'05, 2, 60-65 (2005).

[14] Burt P.J. and Adelson E.H., "The Laplacian pyramid as a compact image code," IEEE Transactions on Communications, 31(4), 532-540 (1983).

[15] Raol J.R., Taylor \&amp; Francis Group, Boca Raton, London \&amp; New York, 383-384 (2010).

[16] Li S., Kwok J. T., and Wang. Y., "Combination of images with diverse focuses using the spatial frequency," Information Fusion, 2,169-76 (2001).

[17] Patil U. and Mudengudi U., "Image fusion using hierarchical PCA," Proc. of ICIIP, 978-1-61284-861-1/11 (2011).

[18] Stampanoni M., Wang Z., "The first analysis and clinical evaluation of native breast tissue using differential phase contrast mammography," Investigative Radiology, 46(12), (2011). 